\title{
Introduction to the Special Issue on EC'19
}

The ACM Transactions on Economics and Computation (TEAC) is proud to present a special issue with extended versions of a selected set of papers presented at the 20th ACM Conference on Economics and Computation (EC'19). EC'19 was held in Phoenix, AZ, June 24-28, 2019, with Nicole Immorlica and Ramesh Johari serving as program chairs. EC is the leading scientific conference on advances in theory, systems, and applications at the interface of economics and computation. Based on the conference reviews, we as guest editors invited a set of papers that were among the top rated of the over 100 papers accepted for presentation at EC'19; the journal versions of these papers were required to extend upon the conference papers and went through an independent and rigorous review process in a very short time. We are delighted to present the resulting set of six articles, spanning issues from fairness in social choice, incentive alignment, influence maximization, and auction design.

In the first article, Yu Cheng, Zhihao Jiang, Kamesh Mungala, and Kangning Wang consider the fairness of a selected committee through the prism of stability, showing that a stable lottery over committees always exists, and suggest an efficient algorithm for approximately stable lottery.

In the next article, focusing on dichotomous preferences, Haris Aziz, Anna Bogomolnaia, and Hervé Moulin consider three known mechanisms from the literature for choosing a fair mixture, and uncover an intricate tradeoff between their efficiency, fairness, and strategy proofness properties.

In the next article, Jon Kleinberg and Manish Raghavan model the way in which strategic agents may invest effort in gaming a system that evaluates them. The authors consider mechanisms that align their incentives.

In the next article, Grant Schoenebeck and Biaoshuai Tao study the influence maximization problem in undirected networks and prove hardness of approximation for the common linear threshold and independent cascade models.

In the next article, Nick Gravin, Yaonan Jin, Pinyan Lu, and Chenhao Zhang obtain tight approximation guarantees for budget-feasible mechanisms with an additive buyer and propose new deterministic and randomized mechanisms.

In the next article, Evangelia Gergatsouli, Brendan Lucier, and Christos Tzamos ask whether one can construct a truthful mechanism for a welfare maximization problem, given black-box access to an optimization algorithm and priors over types. They provide a negative answer even for very simple problems.

$\mathrm{Hu} \mathrm{Fu}$

Reshef Meir

Guest Editors

\footnotetext{
ACM Reference format: Article 17 (November 2020), 1 page.

https://doi.org/10.1145/3424651

(c) 2020 Copyright held by the owner/author(s).

2167-8375/2020/11-ART17

https://doi.org/10.1145/3424651
}

$\mathrm{Hu} \mathrm{Fu}$ and Reshef Meir. 2020. Introduction to the Special Issue on EC'19. ACM Trans. Econ. Comput. 8, 4,

ACM Transactions on Economics and Computation, Vol. 8, No. 4, Article 17. Publication date: November 2020. 